\title{
Case of pazopanib-induced thyrotoxicosis in a patient with metastatic renal cell carcinoma
}

\author{
Sohei Kuribayashi ${ }^{1}$ Tetsuya Takao ${ }^{1} \cdot$ Yohei Okuda $^{1} \cdot$ Masataka Kawamura $^{1}$. \\ Kosuke Nakano $^{1}$ - Nozomu Kishimoto ${ }^{1} \cdot$ Kentaro Takezawa $^{1}$ - Go Tanigawa ${ }^{1}$. \\ Koichi Tsutahara $^{1} \cdot$ Hirotaka Watanabe $^{2}$ Yutaka Umayahara $^{3} \cdot$ Seiji Yamaguchi $^{1}$
}

Received: 15 January 2017 / Accepted: 22 March 2017 / Published online: 27 March 2017

(C) The Japan Society of Clinical Oncology 2017

\begin{abstract}
Pazopanib is an oral multi-targeted tyrosine kinase inhibitor and has been approved for metastatic renal cell carcinoma and advanced soft tissue sarcoma. To the best of our knowledge, pazopanib-induced thyrotoxicosis has never been reported. The patient was a 68 -yearold woman with renal cell carcinoma and multiple metastases. Three weeks after pazopanib medication, she felt fatigue and palpitation. She was admitted because of liver chemistry abnormalities [AST 723 IU/L (normal range 7-37 IU/L); ALT 953 IU/L (normal range 3-33 IU/L)]. She showed abnormal thyroid function tests with suppressed TSH of $0.0585 \mu \mathrm{IU} / \mathrm{mL}$, and elevated fT4 of $3.38 \mathrm{ng} / \mathrm{dL}$. Ten days after admission, tachycardia appeared and fT3 and fT4 were elevated (fT3 $27.85 \mathrm{pg} / \mathrm{mL}$, fT4 $2.58 \mathrm{ng} / \mathrm{dL}$ ), and TSH was suppressed $(0.0414 \mu \mathrm{IU} / \mathrm{mL})$. Her thyroid ultrasound showed hypervascular thyroid gland without nodules. We diagnosed pazopanib-induced thyrotoxicosis. Because the fT3/fT4 ratio was high (10.8) and the thyroid was enlarged, we thought that her thyrotoxicosis might get worse. She was treated with medication of beta-blockers, hydrocortisone, and potassium iodide and thyrotoxicosis resolved. This report highlights that Pazopanib could induce thyrotoxicosis, and therefore, periodic surveillance of thyroid function is required during pazopanib therapy.
\end{abstract}

Sohei Kuribayashi

s.kuri.317@gmail.com

1 Department of Urology, Osaka General Medical Center, 3-1-56 Bandai Higashi, Sumiyoshi-ku, Osaka 558-8558, Japan

2 Department of Diabetes and Metabolism, Japan Community Health Care Organization Osaka Hospital, Osaka, Japan

3 Department of Diabetes and Metabolism, Osaka General Medical Center, Osaka, Japan
Keywords Pazopanib $\cdot$ Thyrotoxicosis $\cdot$ Renal cell carcinoma

\section{Introduction}

Pazopanib is one of the tyrosine kinase inhibitors (TKI) approved for the treatment of advanced renal cell carcinoma [1]. Pazopanib inhibits vascular endothelial growth factor (VEGF) receptor, platelet-derived growth factor (PDGF) receptor, and C-Kit tyrosine kinase receptors [1].

TKI-induced thyroid dysfunction was reported in 2006 in patients that had gastrointestinal stromal tumors and received sunitinib [2]. However, TKI-induced thyrotoxicosis has rarely reported before. To the best of our knowledge, pazopanib-induced thyrotoxicosis has never been reported. Here, we report a case of pazopanib-induced thyrotoxicosis.

\section{Case report}

The patient was a 68-year-old female who was diagnosed with Stage II renal clear cell carcinoma in 2013 and had a radical nephrectomy. Her past medical history included aortic dissection and ovariectomy. She had a family history of hypertension. Two years after the operation, she was found to have metastasis to the sacrum by follow-up computed tomographic scanning. She then received palliative radiation therapy to sacrum for control of the pain (45 Gy).

After the radiation therapy, she started receiving pazopanib (600 mg per day). Three weeks after the therapy, she discontinued pazopanib because of fatigue and palpitation. She was admitted to our hospital because of liver chemistry abnormalities [AST 723 IU/L (normal range 7-37 IU/L); 
ALT 953 IU/L (normal range 3-33 IU/L)]. She showed abnormal thyroid function tests (TFTs) with suppressed $\mathrm{TSH}$ of $0.0585 \mu \mathrm{IU} / \mathrm{mL}$ (normal range $0.35-4.94 \mu \mathrm{IU} / \mathrm{mL}$ ), and elevated fT4 $3.38 \mathrm{ng} / \mathrm{dL}$ (normal range $0.70-1.48 \mathrm{ng}$ / dL).

On physical examination, her thyroid was enlarged, and her vital signs included temperature of $36.7^{\circ} \mathrm{C}$, heart rate of $115 \mathrm{bpm}$, and blood pressure of $116 / 83 \mathrm{mmHg}$. We diagnosed drug-induced thyroid dysfunction; however, we thought that there was no need for medication at that time. Other adverse events such as hypertension and hand-foot syndrome had not happened.

Ten days after admission, paroxysmal atrial fibrillation appeared. At that time, TFTs showed suppressed TSH of $0.0414 \mu \mathrm{IU} / \mathrm{mL}$, elevated fT4 $2.58 \mathrm{ng} / \mathrm{dL}$, and elevated fT3 $27.85 \mathrm{pg} / \mathrm{mL}$ (normal range 1.71-3.71 pg/mL). Thyroglobulin was elevated at $2716 \mathrm{ng} / \mathrm{mL}$ (normal range $<33.7 \mathrm{ng}$ / $\mathrm{mL}$ ), and antithyroglobulin was slightly elevated at $33 \mathrm{IU} /$ $\mathrm{mL}$ (normal range $<28 \mathrm{IU} / \mathrm{mL}$ ), whereas antithyroid peroxidase and thyroid-stimulating hormone receptor antibody were not detected. Her thyroid ultrasound showed hypervascular thyroid gland without nodules.

Because the fT3/fT4 ratio was high (10.8) and the thyroid was enlarged, we thought that her thyrotoxicosis was due to Graves' disease. She was treated with beta-blockers, hydrocortisone, and potassium iodide. She was not treated with propylthiouracil and thiamazole because of her liver chemistry abnormalities.

The day after the treatment, the atrial fibrillation disappeared, and her heart rhythm returned to sinus rhythm. She was discharged from our hospital 20 days after admission. One month after discharge, TSH was elevated, and fT3, fT4, AST, and ALT had returned to normal range (Fig. 1). Ultrasound showed shrinkage of thyroid. Three months after discharge, TFTs showed hypothyroidism, and L-thyroxine was started. She have taken L-thyroxine until now.
We proposed to her another TKI or switch to mammalian target of rapamycin (mTOR) inhibitor, but she selected observation therapy. She remains alive and has had stable disease for 1 year.

\section{Discussion}

TKIs have been approved for treatment of metastatic renal cell carcinoma in Japan, the US, and European countries. Pazopanib, an oral angiogenesis inhibitor targeting VEGF, PDGF, and c-Kit, is an effective treatment for advanced renal cell carcinoma [1]. TKIs have been associated with thyroid dysfunction, mainly hypothyroidism. TKI-induced thyroid dysfunction was reported in 2006 in patients that had gastrointestinal stromal tumors and received sunitinib [2]. Pazopanib-induced hypothyroidism was recorded in $12 \%$ of renal cell carcinoma patients [3]. Although TKIinduced thyroid dysfunction has been reported, TKIinduced thyrotoxicosis has rarely been described. Sorafenib and sunitinib-induced thyrotoxicosis have been reported [4, 5]. Recent reports show that sunitinib-induced thyrotoxicosis has been recorded $3 \%$ of renal cell carcinoma [6], and chronic thyroiditis may be the risk factor [7]. Pazopanibinduced thyrotoxicosis has never been reported before. This is the first reported case of thyrotoxicosis induced by pazopanib. The mechanism of thyrotoxicosis was thought to be due to destructive thyroiditis [8]. Although several possible mechanisms have been suggested for TKI-induced thyroiditis, such as blocking of iodine uptake [9], autoimmune mechanism [10], and VEGF inhibition [11], the mechanism is still unclear.

Destructive thyroiditis initially presents as transient thyrotoxicosis with decreased radionuclide uptake, followed by a hypothyroid phase. In our case, thyroglobulin was extremely high, and TFTs were improved rapidly and TSH
Fig. 1 TFTs results during the treatment

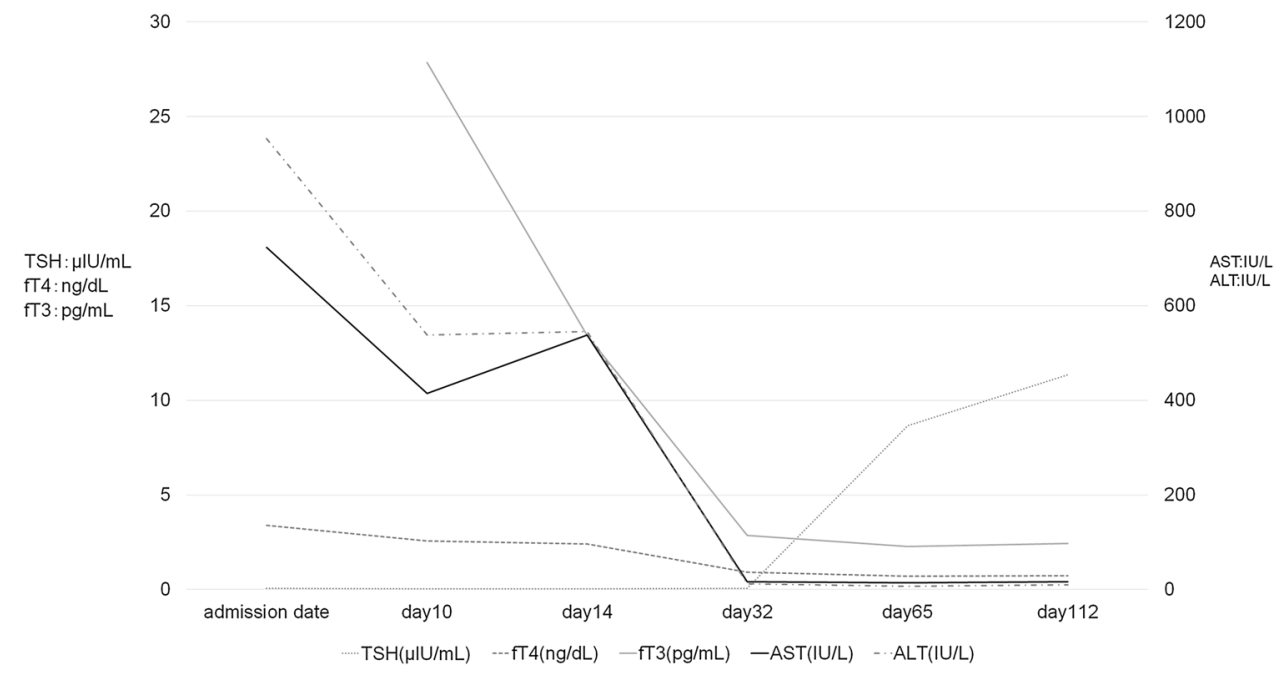

30 
was elevated. This course accords with destructive thyroiditis. Unfortunately, we did not perform thyroid scintigraphy for distinguishing Graves' disease from destructive thyroiditis. If we performed such a thyroid scan, the findings might be useful for determining the mechanism of thyrotoxicosis.

At first, the patient's vital signs were stable without tachycardia, and her treatment with pazopanib was stopped. However, her thyroid dysfunction worsened and paroxysmal atrial fibrillation appeared. Although treatment of TKI-induced thyrotoxicosis is not always required, our case needed to be treated with corticosteroid and beta-blockers, because fT3-to-fT4 ratio was very high. The fT3-to-fT4 ratio was useful for differentiating between Graves' disease from destructive thyroiditis $[12,13]$. Recent report shows that this ratio $>4.4$ confirms the diagnosis of Graves' disease [13]. At that time, we thought that thyrotoxicosis was due to Graves' disease and we started therapy. Because propylthiouracil and thiamazole should be taken carefully for patients with liver dysfunction, we did not use these two drugs in initial treatment.

TKI-induced hypothyroidism is almost reversible. However, in some case, it can be irreversible. It has been reported that shrinkage of thyroid volume might be a potential marker of irreversible thyroid dysfunction [14]. In our case, thyroid volume decreased 30\% after the treatment of pazopanib.

Pazopanib is metabolized in the liver, and our patient had liver dysfunction, so impaired metabolism might have resulted in an increase of the serum concentration of pazopanib. The incidence of ALT elevation in pazopanib ranges from 46 to $60 \%$ [15], so pazopanib-induced thyroid dysfunction may be exacerbated in patients with liver chemistry abnormalities. Thus, regular surveillance of thyroid function is essential during pazopanib therapy. If liver abnormalities appear, TFTs should be performed after the cessation of pazopanib.

In conclusion, pazopanib can induce thyrotoxicosis, which can become worse after the cessation of pazopanib. Further data should be obtained to determine the mechanism of pazopanib-induced thyrotoxicosis.

\section{Compliance with ethical standards}

Conflict of interest The authors declare that they have no conflict of interest.

Research involving human participants and/or animals For this type of study, formal consent is not required.
Informed consent Informed consent was obtained from the patient including this report.

\section{References}

1. Sternberg CN, Davis ID, Mardiak J et al (2010) Pazopanib in locally advanced or metastatic renal cell carcinoma: results of a randomized phase III trial. J Clin Oncol 28:1061-1068

2. Desai J, Yassa L, Marqusee E et al (2006) Hypothyroidism after sunitinib treatment for patients with gastrointestinal stromal tumors. Ann Intern Med 145:660-664

3. Motzer RJ, Hutson TE, Cella D et al (2013) Pazopanib versus sunitinib in metastatic renal-cell carcinoma. N Engl J Med 369:722-731

4. Haraldsdottir S, Li Q, Villalona-Calero MA et al (2013) Case of sorafenib-induced thyroid storm. J Clin Oncol 31:e262-e264

5. Sakurai K, Fukazawa H, Arihara Z et al (2010) Sunitinibinduced thyrotoxicosis followed by persistent hypothyroidism with shrinkage of thyroid volume. Tohoku J Exp Med 222:39-44

6. Jazvić M, Prpić M, Jukić T et al (2015) Sunitinib-induced thyrotoxicosis - a not so rare entity. Anticancer Res 35:481-485

7. Daimon M, Kato T, Kaino W et al (2012) Thyroid dysfunction in patients treated with tyrosine kinase inhibitors, sunitinib, sorafenib and axitinib, for metastatic renal cell carcinoma. Jpn J Clin Oncol 42:742-747

8. Grossmann M, Premaratne E, Desai J et al (2008) Thyrotoxicosis during sunitinib treatment for renal cell carcinoma. Clin Endocrinol (Oxf) 69:669-672

9. Mannavola D, Coco P, Vannucchi G et al (2007) A novel tyrosine-kinase selective inhibitor, sunitinib, induces transient hypothyroidism by blocking iodine uptake. J Clin Endocrinol Metab 92:3531-3534

10. Alexandrescu DT, Popoveniuc G, Farzanmehr H et al (2008) Sunitinib-associated lymphocytic thyroiditis without circulating antithyroid antibodies. Thyroid 18:809-812

11. Kamba T, McDonald DM (2007) Mechanisms of adverse effects of anti-VEGF therapy for cancer. Br J Cancer 96:1788-1795

12. Izumi Y, Hidaka $\mathrm{Y}$, Tada $\mathrm{H}$ et al (2002) Simple and practical parameters for differentiation between destruction-induced thyrotoxicosis and Graves' thyrotoxicosis. Clin Endocrinol (Oxf) 57:51-58

13. Sriphrapradang C, Bhasipol A (2016) Differentiating Graves' disease from subacute thyroiditis using ratio of serum free triiodothyronine to free thyroxine. Ann Med Surg (Lond) 10:69-72

14. Rogiers A, Wolter P, Op de Beeck K (2010) Shrinkage of thyroid volume in sunitinib-treated patients with renal-cell carcinoma: a potential marker of irreversible thyroid dysfunction? Thyroid 20:317-322

15. Powles T, Bracarda S, Chen $M$ et al (2015) Characterisation of liver chemistry abnormalities associated with pazopanib monotherapy: a systematic review and meta-analysis of clinical trials in advanced cancer patients. Eur J Cancer 51:1293-1302 